\title{
Performance Analysis of a System that Identifies the Parallel Modules through Program Dependence Graph
}

\author{
Shanthi Makka \\ Department of Computer Science and Engineering, JRE Group of Institutions, Greater Noida, 201310, India \\ E-mail: Shanthi.makka@gmail.com \\ Dr. B.B.Sagar \\ Department of Computer Science and Engineering, BITs, Ranchi(Noida Campus201301, India \\ E-mail: drbbsagar@gmail.com
}

Received: 02 March 2017; Accepted: 08 June 2017; Published: 08 September 2017

\begin{abstract}
We have proposed a new approach to identify segments, which can be executed simultaneously, or coextending to achieve high computational speed with optimized utilization of available resources. Our suggested approach is divided into four modules. In first module we have represented a program segment using Abstract Syntax Tree (AST) along with an algorithm for constructing AST and in second module, this AST has been converted into Program Dependence Graph (PDG), the detailed approach has been described in section II, The process of construction of PDG is divided into two steps: First we construct a Control Dependence Graph (CDG, In second step reachability definition algorithm has been used to identify data dependencies between the various modules of a program by constructing Data Dependence Graph (DDG). In third module an algorithm is suggested to identify parallel modules, i.e., the modules that can be executed simultaneously in the section III and in fourth module performance analysis is discussed through our approach along with the computation of time complexity and its comparison with sequential approach is demonstrated in a pictorial form.
\end{abstract}

Index Terms-Abstract Syntax Tree, Program Dependence Graph, Control Dependence Graph, Data Dependence Graph, Performance Analysis, Parallel Modules.

\section{INTRODUCTION}

Despite Moore's law [3], uniprocessor clock speeds have now stalled, rather than using single processors running at ever-higher clock speeds, and drawing ever increasing amounts of power, even consumer laptops, tablets and desktops now have dual, quad or hexa-core processors. Job Scheduling on parallel computer [21] or parallel machines has been an imminent topic in the past couple of decades. Many researchers are working to invent optimization techniques for scheduling multiple jobs on parallel machines. So researchers started thinking parallel [13] to achieve good performance of applications and co up with technological growth reflecting in day-to-day life. One approach is to parallelize a program [9] is to rewrite it from scratch. However, the most common way is to parallelize a program incrementally, one piece at a time. Each small step can be seen as a behavior preserving transformation, i.e., a refactoring Programmer prefer this approach [4] because it is safer; they prefer to maintain a working, deployable version of the program. Also, the incremental approach is more economical than rewriting. For our approach we consider input as Abstract Syntax Tree i.e., The Abstract Syntax Tree (AST) [5] is a representation of the source code that is commonly used in compilers. It gives complete representation of the source code in the sense that it is possible to re generate an equivalent version of the original source code from an AST. The only things that are not modeled by an AST are spaces, blank lines and comments. The AST is closely related to the parse tree and hence to the formal grammar of the programming language. The only difference with the parse-tree is that the AST usually removes useless constructs, such as useless parentheses. The rules on what and how useless constructs are removed are not clearly defined and vary from one implementation to another.

The PDG [19] is constructed for the AST in the preceding step, which procreate perspicuous twain the data and control dependences for each operational statement in a program. Data dependence graphs have sustained some optimizing compilers [11] with a definite effigy of the definition-use relationships inherently commenced in a source program [6,7]. A control flow graph [8] is a conventional embodiment for the control flow interconnections of a program; the control conditions on which an operation depends can be described in a graphical form. The program dependence 
graph extraordinarily denotes both the essential data relationships, as present in the data dependence graph, and the important control relationships, without the unwanted sequencing present in the control flow graph. These essential dependences determine the necessary sequencing between operations, producing potential parallelism. Approachability of statements in a program can be determined by using reachability definition algorithm and also demonstrated through an example and Finally a new approach where even topological sort is used to find nearest predecessor which has been demonstrated algorithmically as well as with example and its computational time is also calculated and graphically depicted.

\section{CONSTRUCTION OF PDG}

The primary dominion of graph transformations [14] in inured is that they effete a mannered and mathematical model that capitulate for divergent cursory fortuitous upon abilities, umbrella of the aptitude prone to a given transfiguration is remedy. Flow analysis is a technique of analysis of data and control flows of a source program. In object-oriented programming languages [1], preconditions [15] can be established by the data flow in source program i.e., the extracted method is acknowledged with at most one result and the control flow in a program conciliate whether the method can be extracted at all or not i.e., it has to verify that there must be a unique entry and unique exit precondition scrap. Program Dependence Graph (PDG) is a figurative illustration [2] of a program moiety, which has a docility of representing both control and data dependences between different segments of program. Control dependences mediates next instruction to be executed and data dependences pageant the value ascribe to variable ' $a$ ' in statement ' $\mathrm{X}$ ' is recycled in other sections in a program. The construction of PDG consists of two steps, initially construct Control Dependence Graph (CDG) for an Abstract Syntax Tree (representation of a program segment) and secondly incorporate data dependencies to CDG using reachability algorithm.

\section{A. Abstract Syntax Tree (AST)}

An AST is a non-linear data structure, hierarchical representation of the abstract syntactic anatomy [5] of source code drafted in a programming language. Each node of the tree signifies a construct occurring in the source code or every individual statement represented as a node and it is widely targeted in syntax analyzer phase of a compiler. In other words it is basically represents the structure of code. Abstract syntax trees are data structures extensively employed in compiler design, due to property of personify the representation of program code. The generation of AST is an output of syntax analyzer, which is a second phase of compilation. It recurrently oblige as an intermediate representation of the program through multiple phases that the compiler expects, and has a great role in the determination of targeted production of the compiler.
It provides abstract view of a given source code and first node (root) is main_node, it has eight Children to represent S1 to S6 statements, Predicate P1 and statement S10. The while loop has three children one for condition inside while, second for if_else and one for S9. The conditional construct if_else has further has three links one for it's own condition, one for if clause and one for else_clause. The construction of AST is shown here for the given below segment.

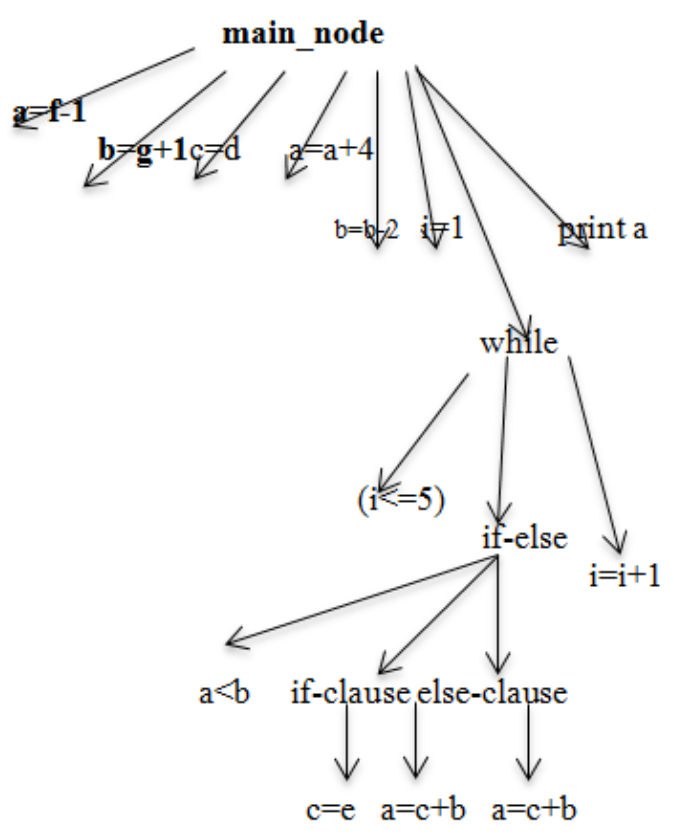

Fig.1. Program with AST

$$
\begin{aligned}
& \text { Program segment: } \\
& \text { S1: } \mathrm{a}=\mathrm{f}-1 \\
& \text { S2: } \mathrm{b}=\mathrm{g}+1 \\
& \text { S3: } \mathrm{c}=\mathrm{d} \\
& \text { S4: } \mathrm{a}=\mathrm{a}+4 \\
& \text { S5: } \mathrm{b}=\mathrm{b}-2 \\
& \text { S6: } \mathrm{i}=1 \\
& \text { P1: while }(\mathrm{i}<=5) \\
& \text { P2: if }(\mathrm{a}<\mathrm{b}) \text { then } \\
& \text { S7: } \quad \mathrm{c}=\mathrm{e} \\
& \text { S8: } \quad \mathrm{a}=\mathrm{c}+\mathrm{b} \\
& \text { S9: } \quad \mathrm{i}=\mathrm{i}+1 \\
& \text { S10: print } \mathrm{a}
\end{aligned}
$$

\section{B. Construction of $C D G$}

CDG is Control Dependence Graph, which reflects control dependences between different segments of a program. If a statement ' $A$ ' has control dependence on statement ' $\mathrm{B}$ ', then ' $\mathrm{B}$ ' has to be executed priory as compared to ' $A$ '. In this section, we have demonstrated our approach with an algorithm and as well as with an example. The CDG construction takes Abstract Syntax Tree (AST) as an input and Produces Control Dependence Graph (CDG) as an output. To maintain simplicity in explanation, we consider structured control statements i.e., while and if_else, simple assignment statements, unconditional transfer of control statements 
i.e., goto statements and so on. To implement our approach we used data structure Stack and it's operations PUSH and POP to insert and delete an element from the stack to maintain nesting and control dependences between the modules of a program. To have access on each and every statements of AST, preorder traversal (root, left, right) is done. Algorithm begins with construction of entry node, which denotes entire process is created and placed in STACK, subsequently nodes are added to the STACK, the children of the nodes are at the top of the STACK. Once innermost statements on the top of the STACK get evaluated, then next level of the statement will be evaluated. For example if_else statement in placed inside the while loop, node will be added as active or live for if_else, once it is evaluated then control moves to the while statement.

Whenever there is a conditional constructs in program, one region node is created with the name $\mathrm{Ri}$, it has further child link to predicate node $(\mathrm{Pi})$, which in turn denotes the conditional statement. As Pi is a conditional statement, it has two children links one for true and other for false, again two region (body) nodes will be created for true and false clauses of predicate node, under these region nodes the corresponding statements will be evaluated. Through backward arrows repetition of statements under looping constructed are handled and these backward arrows will be connected to the entry of that region node $(\mathrm{Ri})$.

\author{
Algorithm: \\ Algorithm CDG_construction( $\mathrm{T}, \operatorname{root}[\mathrm{T}])$ \\ // $\mathrm{T}$ is abstract syntax tree; root $[\mathrm{T}]$ is address of $\mathrm{T}$. \\ //This algorithm takes AST of a program $\mathrm{S}$ as input and \\ produces $C D G$ as output for the program $S$. \\ //For implementation the PUSH and POP operations on \\ the data structure STACK (ST) is performed. \\ //main_node is nothing but root[T] \\ \{ \\ while ( nodes are present in AST) do \\ \{ \\ node=call CDG_Insert_Node( ); \\ switch( node) \\ \{ \\ case main_node: \\ \{ \\ node=call CDG_Insert_Node(entry,live ); \\ consider node as root of $\mathrm{CDG}$ \\ PUSH(ST,node); \\ Create an exit node for latter use; \\ \} \\ case '=': \\ \{ \\ node=call CDG_Insert_Node(statement, live ); \\ \} \\ case 'while': \\ \{//beginning of while \\ node=call CDG_Insert_Node(head, live); \\ PUSH(node); \\ node=call CDG_Insert_Node(predicate,node ); \\ node $=$ call CDG_Insert_Node $($ body,predicate $)$;
}

\author{
PUSH(node); \\ // end of while; condition id false \\ node $=\mathrm{POP}(\mathrm{ST})$
}

solve the statement in 'node' node $=\mathrm{POP}(\mathrm{ST})$

this will be the header node, i.e. the body of while loop is executed

node=call CDG_Insert_Node(statement followed by while,live );

consider node as Top of the stack

\}

case 'if_else':

\{

node=call CDG_Insert_Node(predicate,live ); PUSH(ST,node);

Begin if_clause(true)

node=call CDG_Insert_Node(body,live );

Begin else_clause(flase) PUSH(ST,node);

node=call CDG_Insert_Node(body,live );

End of if_else PUSH(ST, node);

Node $=$ POP $($ ST)

Solve the statement in node

node=call CDG_Insert_Node(statement followed by ifelse,live );

consider node as Top of the stack

end of if_clause or end of else_clause

list out all the unresolved nodes

node=POP(ST); \}

case 'structured control transfer':

\{

node=call CDG_Insert_Node(statement,live );

find out the follow information and update STACK

Add special flow and dependence edge \}

case 'goto': \{

node=call CDG_Insert_Node(statement,live );

update label table

set flag to true $\}$

case 'label':

\{

node=call CDG_Insert_Node(label body,live );

PUSH(ST,node);

update label table

\}) \}

node=call CDG_Insert_Node(exit,live );

\}

In our algorithm, we have used while loop at the beginning to consider every node in AST, at the same time we have created exit node for later use. Procedure like CDG_Insert_Node( ) has two parameters $\mathrm{X}, \mathrm{Y}$ is used to create a now node for $\mathrm{X}$ which will be made as child to $\mathrm{Y}$, i.e., it adds an edge between $\mathrm{X}$ and $\mathrm{Y}$. When an algorithm finds end of any looping construct, then it pops all the statements in side that loop and inserts a backward arrow to region node of that loop. When it reaches to end of if_else statement in AST, algorithm pops all the statements under if_else and makes followed statement as top of the stack if present. Otherwise it reaches to exit node. 


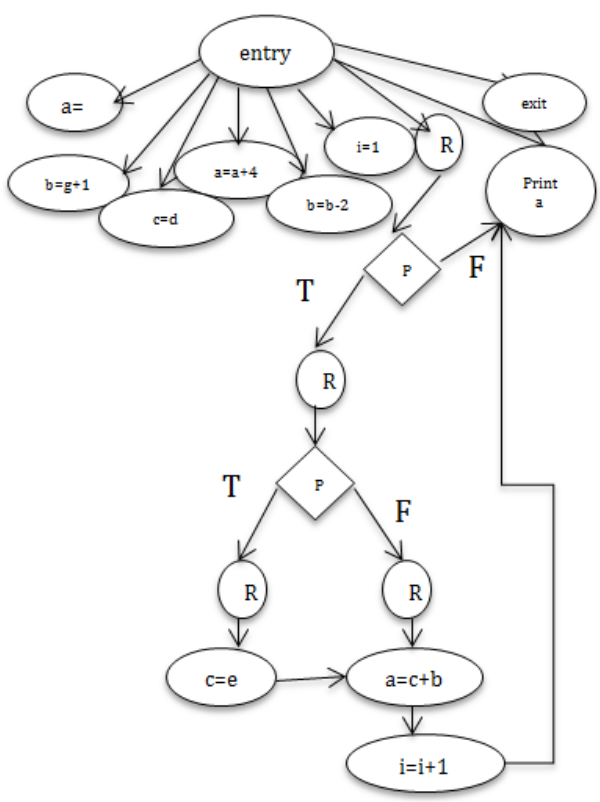

Fig.2. Construction of CDG

\section{Construction of DDG}

DDG is Data Dependence Graph, which depicts the data dependences between segments of a program. If there is a data dependency [12] from a block ' $\mathrm{X}$ ' to block ' $\mathrm{Y}$ ', then the segment represented by ' $\mathrm{X}$ ' assigns some value to a variable, which will be used at the segment represented by ' $Y$ '. Here we have chosen a reachability algorithm to identify data dependences between statements or modules. Preferably we have selected blocks [18] due to clumsiness created due to individual statements consideration. For every block we have created four sets of values [8], GEN[B] is set of variables their values are currently used in block B, prior to any definition of that variables. KILL[B] is set of variables assigns some values in $\mathrm{B}$, prior to any use of that variable in B. OUT [B] is set of variable active after that block B or OUT[B]= $\mathrm{IN}[\mathrm{S}]$, where $\mathrm{S}$ is a successor of $\mathrm{B}$. $\mathrm{IN}[\mathrm{B}]$ is set of variable active at the beginning of that block B. IN $[\mathrm{B}]=\mathrm{GEN}[\mathrm{B}]$ U (OUT[B]-KILL[B]). The DEF,KILL,IN and OUT are to be calculated for every statement in the program and OUT set has to be computed for predicate or region nodes in CDG.

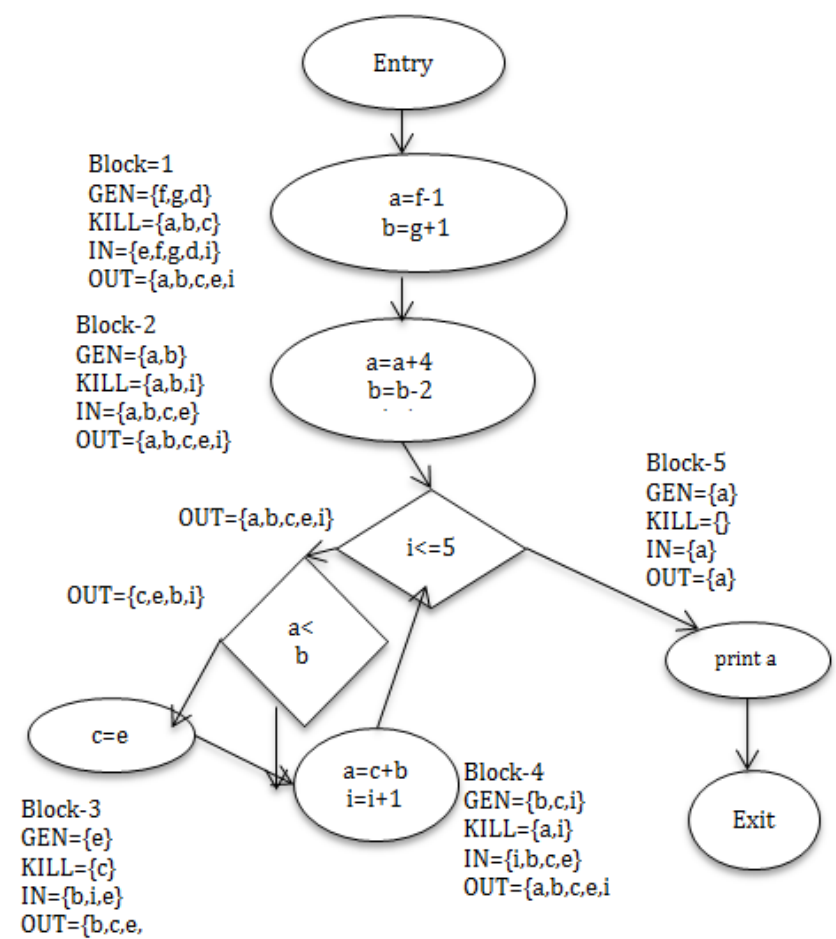

Fig.3. Construction of DDG

In our example, GEN of first block is $\mathrm{f}, \mathrm{g}$ and $\mathrm{d}$, because $f, g$ and $d$ are used on the right hand side of the three assignment statements before they are assigned any values. In fact, they are not assigned any values at all. Whereas, in the case of second block, a and b are used; before they are assigned a values. So, GEN will be a and $b$ and KILL set do not contain a, b because of their accessibility before an assignment, but while exiting this block $\mathrm{a}$ and $\mathrm{b}$ assigned new values which are not used in that block i.e., KILL set also contains a and b. Even in case of simple assignment statement this is true, i.e., e is generated where as c is killed. As discussed earlier for predicate nodes only OUT sets will be calculated, for the first predicate node $(i<=5)$ the OUT set contains a,b,c,e, and $i$ because these variables are active or used in the processing after this block. For every predicate node there are two outgoing lines one for true and other for false. In Block-3, variable ' $e$ ' is used at right hand side, so it is included in GEN set and KILL set contains ' $c$ '; it is assigned a value, which is never used in this block. IN 
sets are calculated by using above said formulae or we can consider only those variables which are active at the beginning of the block. In Block-4, GEN set contains b,c, and $i$ because their values are used in right hand side of a statement where as KILL set contains a and i because these values are not used in the same block. OUT and IN sets are calculated by using same process described in previous step. Finally in last block, only print statement is there, GEN set contains only a and KILL set contains no value because no assignment statements in this block and IN and OUT is same, which contains only a.

After combining above two modules, we have constructed PDG in Fig.4. For predicate nodes we have used diamond symbol and rest of all other purposes oval shape symbol has been used.

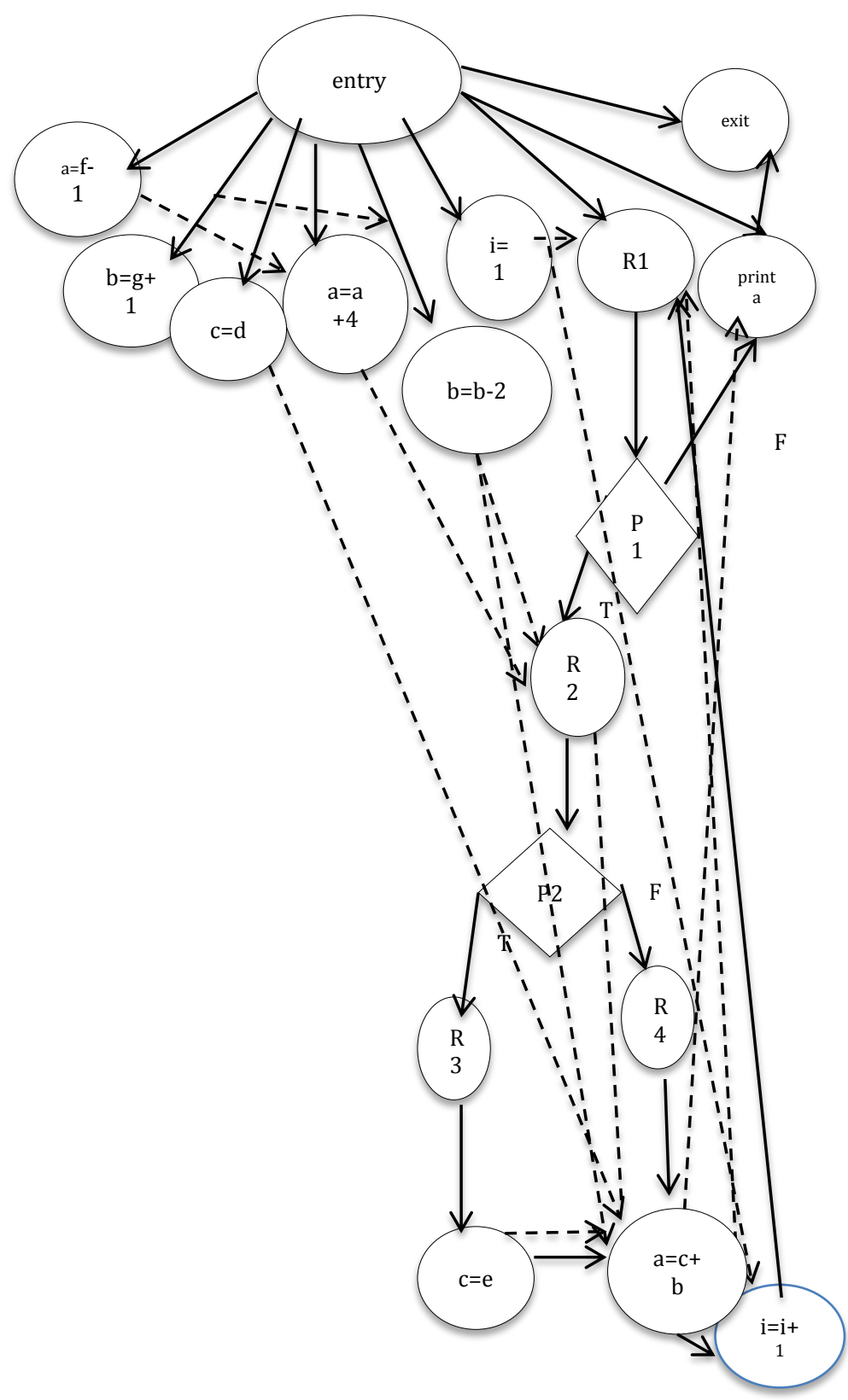

Fig.4. Construction of PDG

$------\rightarrow \quad$ Data dependence lines

Control dependence lines 


\section{IDENTIFICATION OF PARALLEL MODULES}

In our approach, call Reachability Definitions to construct GEN, KILL, IN and OUT sets, then we consider all the vertices in a topological sequence. For every vertex ' $v$ ', find out the nearest predecessor ' $u$ ', if intersection of KILL[u] and IN[v] has some entries i.e., they have dependencies, then $\mathrm{v}$ and $\mathrm{u}$ can not be executed parallel. Otherwise they can be executed parallel. Demonstrated through an example in fig. 5.

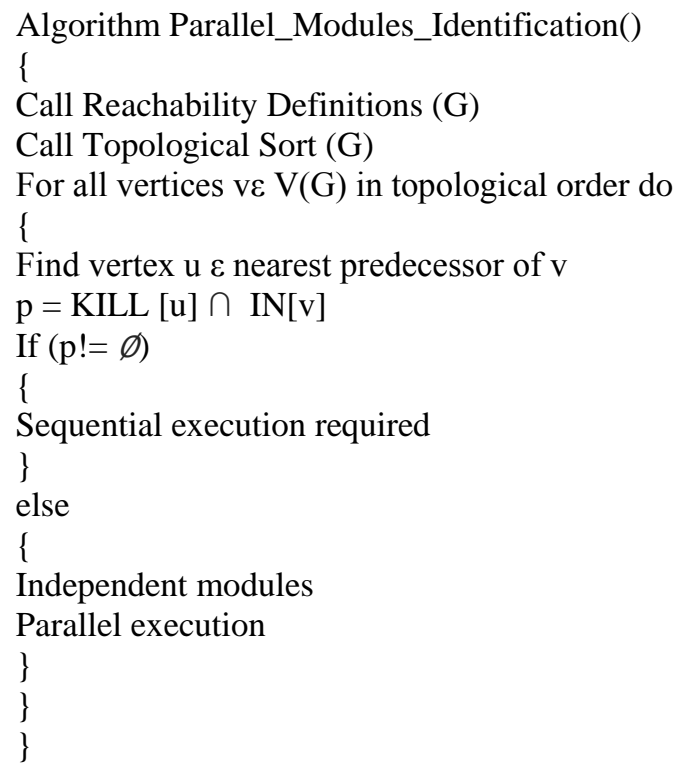

\section{A. Reachability Definitions}

In Graphical representation of data, reachability [20] means path existence between any two vertices of a graph. In our approach discussing about reachability of definitions of a variable means the values assigned to a variable in a particular block is approachable to any other block, i.e., is used by any other variable in other block or vertex. We began with finding out GEN and KILL sets construction, then initialized IN sets of all the vertices to GEN set of that node. We have considered a change variable as flag, if it doesn't change it's value that means final sets for IN and OUT is ready. Till then update IN and OUT sets as mentioned in the algorithm.

Algorithm Reachability Definitions (G)
\{
GEN $[B]=$ set of variables used in block B

$\mathrm{KILL}[\mathrm{B}]=$ set of variables has definitions in block B;

for all blocks in a program do

\{

$\mathrm{IN}[\mathrm{B}]=\mathrm{GEN}[\mathrm{B}]$

\}

change $=0$;

do

\{

for each block B do

\{

if ( block is a predicate node $\|$ region node) then

$\mathrm{OUT}[\mathrm{B}]=\mathrm{OUT}[\mathrm{P}]$, where $\mathrm{P}$ is a predecessor of $\mathrm{B}$ else

\{

// forward flow

$\mathrm{IN}[\mathrm{B}]=$ U OUT[P], where $\mathrm{P}$ is a predecessor of $\mathrm{B}$ OUT[B] $=($ OUT[B]-KILL[B] $)$ し GEN[B];

change $=1$;

// backward flow

OUT[B]= UIN $[S]$, where $\mathrm{S}$ is a successor of B

$\mathrm{IN}[\mathrm{B}]=\mathrm{GEN}[\mathrm{B}]$ LJ (OUT[B]-KILL[B])

change $=1$;

\} while(change);

\}

\section{B. Topological Sort}

A topological sort of a given graph $\mathrm{G}=(\mathrm{V}, \mathrm{E})$ is “ $A$ linear Ordering of vertices [16] such that if there is an edge from $u->_{v}$ then ' $u$ ' precedes ' $v$ ' in the sequence". Many algorithms developed [10] to get topological sequence of a graph. The primary applications or Real word applications are instruction scheduling, ordering of formula cell evaluation when re-computing formula values in spreadsheets, logic synthesis, determining the order of compilation tasks to perform in make files, data serialization, and resolving symbol dependencies in linkers. It is also used to decide in which order to load tables with foreign keys in databases.

\section{Algorithm Topological Sort (G) \{ Call DFS (G);}

Insert nodes traversed through DFS in the descending sequence of finishing times in to a linked list; Return list of nodes of a linked list; \}

Global declaration of $\mathrm{t}=0$;

Algorithm DFS (G)

\{

for each vertex $\mathrm{v} \in \mathrm{V}(\mathrm{G})$ do visit[v]=false; //visit[] is a Boolean field if a vertex visited, then it is true otherwise it is false for each vertex $\mathrm{v} \in \mathrm{V}(\mathrm{G})$ do $\operatorname{if}($ visit $[v]==$ false) then call DFS_VISIT(v);

\}

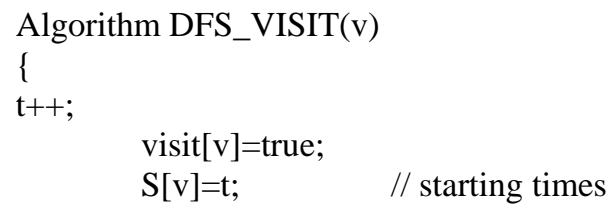

For each vertex $\mathrm{u} \in \operatorname{adj}(\mathrm{v}) \mathrm{do}$ If (visit $[\mathrm{u}]==$ false)<smiles>CC(C)[AsH][AsH2]</smiles>

$\mathrm{t}++$

$\mathrm{F}[\mathrm{v}]=\mathrm{t} ; \quad$ //finishing times 


\section{PERformance AnAlysis}

Performance of a proposed approach can be demonstrated: Reachability algorithm takes $\mathrm{O}\left(\mathrm{n}^{2}\right)$ time, where ' $n$ ' is number of statements in a program, and number of statements is equal to number of vertices in a graph, i.e., time complexity can be expressed as $\mathrm{O}\left(\mathrm{V}^{2}\right)$. Topological sort [17] requires $\mathrm{O}(\mathrm{V}+\mathrm{E})$ time and by considering the vertices in topological sequence, finding out independent modules requires $\mathrm{O}\left(\mathrm{V}^{2}\right)$ time. Total time complexity of our approach is:

$$
\begin{aligned}
\mathbf{T}(\mathbf{n}) \quad & =\mathbf{O}\left(\mathrm{V}^{2}\right)+\mathbf{O}(\mathrm{V}+\mathbf{E})+\mathbf{O}\left(\mathrm{V}^{2}\right) \\
& =\mathbf{2}^{*} \mathbf{O}\left(\mathrm{V}^{2}\right)+\mathbf{O}(\mathrm{V}+\mathbf{E}) \quad \text { if a graphis sparse } \\
& =\mathbf{O}\left(\mathrm{V}^{2}\right.
\end{aligned}
$$

\section{A. Sequential versus Parallel execution}

If a single processor is used for the execution of below program segment, first block requires exactly $m * n$ time, where as second block requires $\mathrm{s}^{*} \mathrm{e}$ time and the last block requires $\mathrm{o}^{*} \mathrm{p}$ time. The total time required is (Linear time complexity)

\section{$\mathbf{T}(\mathbf{n})=\theta(\mathbf{m n}+\mathbf{o p}+\mathbf{s e})$}

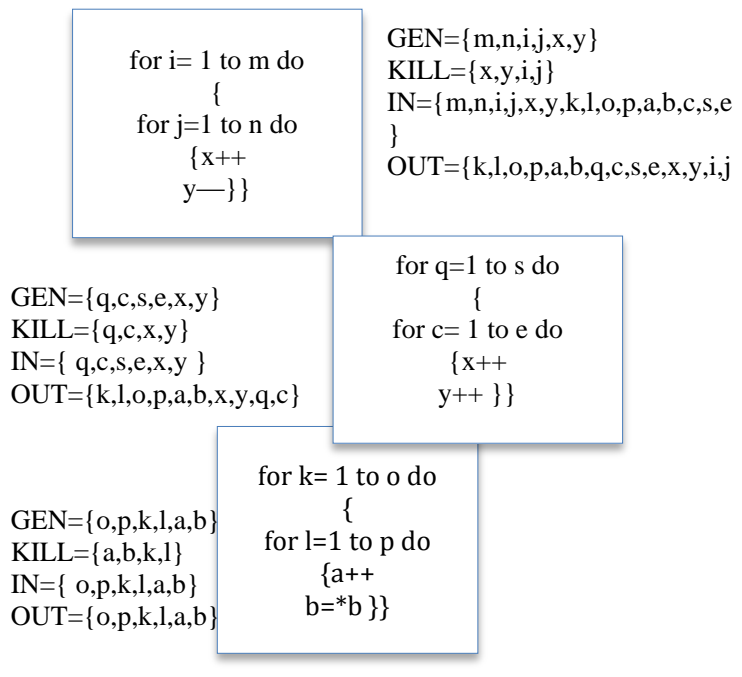

Fig.5. Construction of GEN,KILL,IN,OUT sets

By taking sample data a graph has been plotted with red colored line. For thinking in parallel perspective, find out all four sets for individual modules, i.e., GEN, KILL,
IN and OUT. Make the comparison between IN set data with KILL set of near by predecessor to determine independence modules. If we use two processors for the execution of the same code, it required $\mathrm{s} * \mathrm{e} * \mathrm{f}$ time due to parallel execution of these three independent modules. A graph has been plotted below with blue colored line.

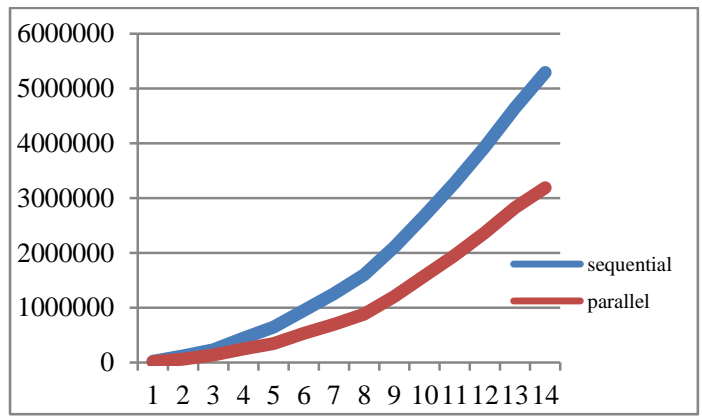

Fig.6. Performance Analysis

\section{B. Speedup}

In parallel computing, the speedup is nothing but ratio between sequential and parallel execution times i.e., it reflects what extent a parallel algorithm is faster than a corresponding sequential algorithm. Speedup is a factor, which improves performance in terms execution after enhancement of resources. In Table 1, observe the data in the column speedup, it varies from 1.5 to 2.0 i.e., The parallel approach is 1.5 to 2 times faster than sequential approach, which has been illustrated through a pictorial representation or in the form of a graph in below figure.

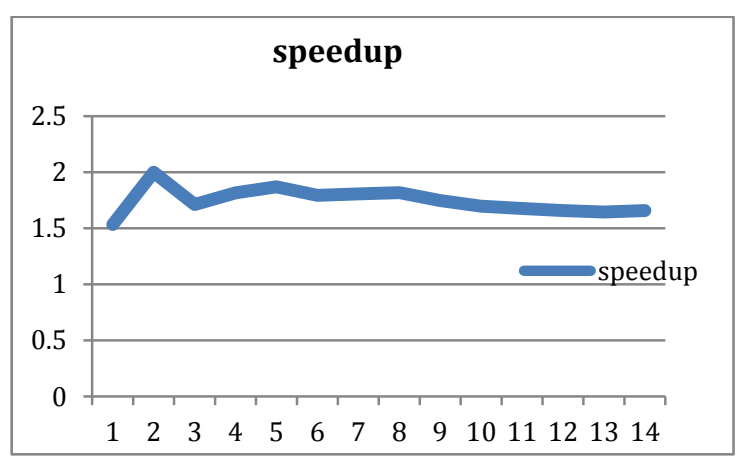

Fig.7. Speedup

In Table 1 data is considered randomly to determine caliber or performance of a suggested system. 
Table 1. Comparison of sequential and Parallel Execution time

\begin{tabular}{|c|c|c|c|c|c|c|c|c|c|c|c|}
\hline $\mathrm{m}$ & $\mathrm{n}$ & $\mathrm{s}$ & $\mathrm{E}$ & $\mathrm{O}$ & $\mathrm{p}$ & $\mathrm{m} * \mathrm{n}$ & $\mathrm{s} * \mathrm{e}$ & $\mathrm{o}^{*} \mathrm{p}$ & Sequential & Parallel & Speedup \\
\hline 100 & 50 & 50 & 200 & 400 & 20 & 5000 & 10000 & 8000 & 23000 & 15000 & 1.533 \\
\hline 200 & 100 & 100 & 400 & 600 & 100 & 20000 & 40000 & 60000 & 120000 & 60000 & 2 \\
\hline 300 & 150 & 200 & 450 & 800 & 120 & 45000 & 90000 & 96000 & 231000 & 135000 & 1.71 \\
\hline 400 & 200 & 300 & 550 & 1000 & 200 & 80000 & 165000 & 200000 & 445000 & 245000 & 1.81 \\
\hline 500 & 250 & 400 & 550 & 1200 & 250 & 125000 & 220000 & 300000 & 645000 & 345000 & 1.86 \\
\hline 600 & 300 & 500 & 700 & 1400 & 300 & 180000 & 350000 & 420000 & 950000 & 530000 & 1.79 \\
\hline 700 & 350 & 600 & 750 & 1600 & 350 & 245000 & 450000 & 560000 & 1255000 & 695000 & 1.80 \\
\hline 800 & 400 & 700 & 800 & 1800 & 400 & 320000 & 560000 & 720000 & 1600000 & 880000 & 1.81 \\
\hline 900 & 450 & 800 & 1000 & 2000 & 450 & 405000 & 800000 & 900000 & 2105000 & 1205000 & 1.74 \\
\hline 1000 & 500 & 900 & 1200 & 2200 & 500 & 500000 & 1080000 & 1100000 & 2680000 & 1580000 & 1.69 \\
\hline 1100 & 550 & 1000 & 1350 & 2400 & 550 & 605000 & 1350000 & 1320000 & 3275000 & 1955000 & 1.67 \\
\hline 1200 & 600 & 1100 & 1500 & 2600 & 600 & 720000 & 1650000 & 1560000 & 3930000 & 2370000 & 1.65 \\
\hline 1300 & 650 & 1200 & 1650 & 2800 & 650 & 845000 & 1980000 & 1820000 & 4645000 & 2825000 & 1.64 \\
\hline 1400 & 700 & 1300 & 1700 & 3000 & 700 & 980000 & 2210000 & 2100000 & 5290000 & 3190000 & 1.65 \\
\hline
\end{tabular}

\section{CONCLUSION}

This paper presents a research plan with an overarching goal to ensure that effectiveness of an approach in identifying parallel segments. While this framework provides new scenario to identify parallel modules. A given application is represented using AST and further it is converted into PDG, and by using reachability definition concept we identified concurrent modules.

\section{FUTURE WORK}

After identifying parallel modules according to our approach, this research plan can be extended for the execution of those modules on heterogeneous parallel architectures according to the topology suggested. This work can further bring in to the parallelization scenario.

\section{REFERENCES}

[1] Mathieu Verbaere, Ran Ettinger and Oege de Moor JunGL. A Scripting Language for Refactoring, 28th International Conference on Software Engineering, pp. 172-181, 2006

[2] Makka Shanthi, and B. B. Sagar. "A New Approach for Optimization of Program Dependence Graph using Finite Automata." Indian Journal of Science and Technology 9.38 (2016).

[3] G. E. Moore, "Readings in Computer Architecture. chapter Cramming more components onto integrated circuits," Morgan Kaufmann Publishers Inc., San Francisco, CA, USA, 2000, pp.56-59.

[4] D. Dig, "A refactoring approach to parallelism," Software, IEEE 28.1 (2011): 17-22.

[5] Baxter, Ira D., et al. "Clone detection using abstract syntax trees." Software Maintenance, 1998. Proceedings, International Conference on. IEEE, 1998.

[6] KUCK, D. J., KUHN, R. H., PADUA, D. A., LEASURE, B., AND WOLFE, M. Dependence graphs and compiler optimizations. In 8th Annual ACM Symposium on Principles of Programming Languages (Williamsburg, VA, Jan. 26-28,1981), ACM, New York, 207-218.

[7] OTTENSTEIN, K. J. Data-flow graphs as an intermediate program form. Ph.D. dissertation, Computer Sciences Dept., Purdue University, Lafayette, IN, August 1978.

[8] AHO, A. V., SETHI, R., AND ULlMAN, J. D. Compilers: Principles, Techniques, and Took. AddisonWesley, 1986. [An alternative reference is this book's predecessor, AHO, A. V. AND ULLMAN, J. D. Principles of Compiler Design. Addison-Wesley, 1977.)

[9] W. F. Opdyke, Refactoring: A Program Restructuring Aid in Designing Object-Oriented Application Frameworks Ph.D. thesis, University of Illinois at Urbana Champaign, 1992.

[10] Pearce, David J., and Paul HJ Kelly. "A dynamic topological sort algorithm for directed acyclic graphs." Journal of Experimental Algorithmics (JEA) 11 (2007): 1-7.

[11] M. Aldinucci, M. Danelutto, P. Kilpatrick, M. Meneghin, and M. Torquati. Accelerating Code on Multi-cores with FastFlow. In Euro-Par, pages 170-181, 2011.

[12] Ferrante, Jeanne, Karl J. Ottenstein, and Joe D. Warren. "The program dependence graph and its use in optimization." ACM Transactions on Programming Languages and Systems (TOPLAS) 9.3 (1987): 319-349.

[13] Jain, Sanjay, and Efim Kinber. "Parallel learning of automatic classes of languages." International Conference on Algorithmic Learning Theory. Springer International Publishing, 2014.

[14] Alhazov, Artiom, Chang Li, and Ion Petre. "Computing the graph-based parallel complexity of gene assembly." Theoretical Computer Science 411.25 (2010): 2359-2367.

[15] Korel, Bogdan. "The program dependence graph in static program testing."Information Processing Letters 24.2 (1987): 103-108.

[16] Meinke, Karl. "Topological methods for algebraic specification." Theoretical computer science 166.1 (1996): 263-290.

[17] Toda, Seinosuka. "On the complexity of topological sorting." Information processing letters 35.5 (1990): 229233.

[18] Tekchandani, Rajkumar, Rajesh Bhatia, and Maninder Singh. "Semantic code clone detection for Internet of Things applications using reaching definition and liveness analysis." The Journal of Supercomputing (2016): 1-28.

[19] Harrold, Mary Jean, Brian Malloy, and Gregg Rothermel. "Efficient construction of program dependence graphs." ACM SIGSOFT Software Engineering Notes. 
Vol. 18. No. 3. ACM, 1993.

[20] Ghiya, Rakesh, and Laurie J. Hendren. "Connection analysis: A practical interprocedural heap analysis for C." International Journal of Parallel Programming 24.6 (1996): 547-578.

[21] Singh, Paramvir. "Hybrid Black Hole Algorithm for BiCriteria Job Scheduling on Parallel Machines." International Journal of Intelligent Systems and Applications 8.4 (2016): 1.

\section{Authors' Profiles}

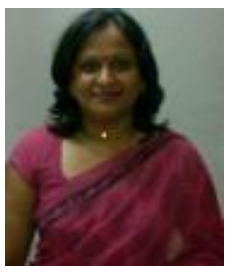

Shanthi Makka, from greater Noida- Uttar Pradesh completed B.tech in Computer Science and Systems Engineering in 2000 from GITAM university, done M.Tech in Computer Science and Engineering and pursuing Ph.D. in Parallel Computing from BITs-Mesra( Noida campus).

Having total experience of 15 years in academics at various places which includes south India, Orissa, Himachal Pradesh and currently working in JRE Group of Institutions since 2014. I am also heading the department of Information Technology at JRE. Publication details (few) are: "A New Approach for Optimization of Program Dependence Graph using Finite Automata." Indian Journal of Science and Technology 9.38 (2016), "Simulation of a Model for Refactoring Approach for Parallelism Using Parallel Computing Tool Box." Proceedings of First International Conference on Information and Communication Technology for Intelligent Systems: Volume 2. Springer International Publishing, 2016, "The Program Dependence Graph and its applications in refactoring ", paper has been ACCEPTED and registered in International Conference on Communication and Networks (COMNET 2016) and publication in Springer AISC series. Accepted on February, 2016 and "Survey on a new advanced approach for refactoring approach for parallelism using heterogeneous parallel architectures", International Journal of Science and Research(IJSR)-ISSN 23197064, Volume 3, Issue 5, May 2014.

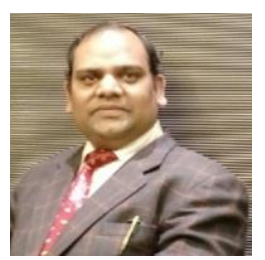

Dr. B. B. Sagar is currently working as an Assistant Professor in Department of CSE, Birla Institute of Technology, Mesra Ranchi and posted at BIT Noida Campus. Received MCA from UPTU and Ph.D. (Computer Science \& Engineering) from SHIATS Allahabad in 2004 and 2011 respectively. Also having 12 years teaching and research experience. Research interests are in Software Reliability, Network Reliability, Parallel Computing and Distributed Computing. A Reviewer of various reputed SCI and Scopus International journals and conferences like Elsevier, IEEE, Springer, Taylor \& Francis, Inderscience (USA) and published more than $\mathbf{4 0}$ research papers in Journal and Conferences of international repute including SCI and Scopus. Chaired many IEEE and other International conference. Also a Professional member of IEEE (USA), IAENG (Hong Kong) and Fellow of IETE (India) and Life member of Vijnana Bharti (India). Got invited in various International summits and conferences as an invited talk and special guest organized by Govt. of India and others. Recently received "Young Scientist Award" at University of Aalborg, Denmark (Europe) in August, 2016.
How to cite this paper: Shanthi Makka, B.B.Sagar, "Performance Analysis of a System that Identifies the Parallel Modules through Program Dependence Graph", International Journal of Intelligent Systems and Applications(IJISA), Vol.9, No.9, pp.37-45, 2017. DOI: 10.5815/ijisa.2017.09.05 